\title{
Testing Criteria and Indicators for Assessing the Sustainability of Forest Management: Genetic Criteria and Indicators
}

\author{
Gene Namkoong, Tim Boyle, Hans-Rolf Gregorius, Hélène Joly, \\ Outi Savolainen, Wickneswari Ratnam and Andrew Young \\ (on behalf of The International Forest Genetics Research Associates)
}

\begin{abstract}
Summary
This working paper contains proposals for specific genetic criteria and indicators (C\&I) which are expected to be part of a more general set of biological C\&I. These proposals are intended for use in guiding tropical forest management but the indicators and verifiers we describe are not in the form of simple prescriptions where a single measurement can be recommended for a single causal effect. Since genetic dynamics operate at a different time and spatial scale than the events that can be observed at the level of forest stand effects, a single forest stand event can have effects on several genetic processes. In addition, the pattern of genetic diversity that has already evolved is due to a balance of several evolutionary forces that operate at different spatial and temporal scales and forest practices would therefore be expected to affect several genetic factors. To provide guidance on what genetic processes may be affected by forest practices, we therefore first describe the factors that affect genetic processes and then provide a matrix of relationships between types of forest level events and genetically significant factors.

Since the intention of the larger project is to steer possible management actions, the report identifies two main concerns of sustainability: first, whether the genetic variation is being maintained, and second, what conservation or enhancement measures can be effective. We state one criterion and for each of these concerns, sets of indicators are defined that would address the issue of sustainability. For each indicator, sets of verifiers are provided which differ in the biologically relevant feature they measure or in the precision and technical facilities they require.

Finally, the need for rapid assessment and precision under difficult field conditions requires research and development of efficient direct and surrogate measures of the genetic resource. We therefore include recommendations for short- and medium term research that would improve the scientific value, cost-effectiveness, ease of use, and further development of genetic criteria and indicators.
\end{abstract}

CENTER FOR INTERNATIONAL FORESTRY RESEARCH

office address: Jalan CIFOR, Situ Gede, Sindangbarang, Bogor 16680, Indonesia

mailing address: P.O. Box 6596 JKPWB, Jakarta 10065, Indonesia

tel.: +62 (251) 622622 fax: +62 (251) 622100

email: cifor@cgnet.com WWW: http://www.cgiar.org/cifor 


\section{The CGIAR System}

The Consultative Group on International Agricultural Research (CGIAR) is an informal association of 41 public and private sector donors that supports a network of sixteen international agricultural research institutes, CIFOR being the newest of these. The Group was established in 1971. The CGIAR Centers are part of a global agricultural research system which endeavour to apply international scientific capacity to solution of the problems of the world's disadvantaged people.

\section{CIFOR}

CIFOR was established under the CGIAR system in response to global concerns about the social, environmental and economic consequences of loss and degradation of forests. It operates through a series of highly decentralised partnerships with key institutions and/or individuals throughout the developing and industrialised worlds. The nature and duration of these partnerships are determined by the specific research problems being addressed. This research agenda is under constant review and is subject to change as the partners recognise new opportunities and problems. 
CENTER FOR INTERNATIONAL FORESTRY RESEARCH

office address: Jalan CIFOR, Situ Gede, Sindangbarang, Bogor 16680, Indonesia mailing address: P.O. Box 6596 JKPWB, Jakarta 10065, Indonesia

tel.: +62 (251) 622622 fax: $+62(251) 622100$

email: cifor@cgnet.com

$W W W:$ http://www.cgiar.org/cifor 


\title{
Testing Criteria and Indicators for Assessing the Sustainability of Forest Management:Genetic Criteria and Indicators ${ }^{1}$
}

\author{
Gene Namkoong, Tim Boyle ${ }^{2}$, Hans-Rolf Gregorius, Hélène Joly, Outi Savolainen, \\ Wickneswari Ratnam and Andrew Young
}

\begin{abstract}
Summary
This working paper contains proposals for specific genetic criteria and indicators (C\&I) which are expected to be part of a more general set of biological C\&I. These proposals are intended for use in guiding tropical forest management but the indicators and verifiers we describe are not in the form of simple prescriptions where a single measurement can be recommended for a single causal effect. Since genetic dynamics operate at a different time and spatial scale than the events that can be observed at the level of forest stand effects, a single forest stand event can have effects on several genetic processes. In addition, the pattern of genetic diversity that has already evolved is due to a balance of several evolutionary forces that operate at different spatial and temporal scales and forest practices would therefore be expected to affect several genetic factors. To provide guidance on what genetic processes may be affected by forest practices, we therefore first describe the factors that affect genetic processes and then provide a matrix of relationships between types of forest level events and genetically significant factors.

Since the intention of the larger project is to steer possible management actions, the report identifies two main concerns of sustainability: first, whether the genetic variation is being maintained, and second, what conservation or enhancement measures can be effective. We state one criterion and for each of these concerns, sets of indicators are defined that would address the issue of sustainability. For each indicator, sets of verifiers are provided which differ in the biologically relevant feature they measure or in the precision and technical facilities they require.

Finally, the need for rapid assessment and precision under difficult field conditions requires research and development of efficient direct and surrogate measures of the genetic resource. We therefore include recommendations for short-and medium term research that would improve the scientific value, cost-effectiveness, ease of use, and further development of genetic criteria and indicators.
\end{abstract}

\section{Introduction}

Genetic variation is required for species to have successfully met the challenges of the past and to survive and reproduce in the current array of conditions they face. Its conservation is also a necessary precondition for the future evolution and adaptability of local populations and of the entire species. Thus, it is both an element of the biodiversity we value and is also a necessary element in the maintenance of all other levels of biodiversity that we value for their existence and utility. However, genetic variation is difficult to measure directly and is often cryptic in its effects on population and ecosystem dynamics. Hence its loss is easy to ignore - until it is too late to restore and to enable ecosystems to sustain themselves by adaptation to changing environments. Genetic erosion ultimately induces species extinctions and ecosystem loss, and forecloses development options for economic gain and ecological restoration.

To avoid extinctions and to maintain or enhance the levels of genetic variation and its distribution among individuals, populations, and species, requires conserving or managing the dynamic forces of evolution largely at the species level. These include mutation, selection, drift and migration, as mediated through the mating system. These forces are not entirely independent of ecological factors which influence them and

1 Within the context of CIFOR's project on “Assessing the Sustainability of Forest Management: Testing Criteria and Indicators", a workshop was organized by The International Forest Genetics Research Associates, at the behest of Dr. Ravi Prabhu, the project leader, in West Java, Indonesia from 8-12, April, 1996

2 Corresponding author. Center for International Forestry Research (CIFOR), P.O. Box. 6596 JKPWB Jakarta 10065, Indonesia. 
which are influenced by genotypically determined effects, but genetic dynamics are not identical with ecosystem dynamics and require separate consideration. Furthermore, these genetic forces are not entirely independent in their effects among each other and the patterns of genetic variation found in any species are the result of their joint effects. Nevertheless, these forces can be separated as factors, can be measured, and therefore can be useful for understanding and estimating the dynamics of change. While we assert no priority for genetic vs. ecological forces or measures, we emphasize their differences and the necessity to estimate different threats and influences. Practical applications of criteria and indicators may require that one serve as a surrogate for the other but they should not be confused by forest managers as being identical.

We emphasize that the conservation of evolutionary processes is a necessary biological criterion by which the sustainability of forests can be judged and we use genetic indicators of those processes to judge the sustainability of those evolutionary processes. To conserve those processes, we define four genetic indicators that are necessary for sustainability. For each indicator, we list several ways to measure threats to those processes that form a tool kit of verifying devices for the forest manager. Some of the verifiers are precise but perhaps expensive or difficult to use, while others are less precise but may be quicker or easier to use.

We are encouraged in our approach by the report of the recently concluded first phase of the CIFOR project on testing criteria and indicators for sustainable forest management (Prabhu et al. 1996). The report documents the current lack of suitable criteria and indicators for assessing impacts on biodiversity, at all levels of the hierarchy, and stresses the urgent need to address this weakness. It also suggests that a tool-box approach to developing and using criteria and indicators for sustainable forest management would have the highest utility for potential user groups.

\section{Effects of Forest Processes on Genetic Dynamics}

As a result of forest management practices or as the unintended side effects of climate change and accidents, changes in genetic variation that follow from such interventions are attributable to changes in the basic evolutionary processes. These processes are; 1 ) random genetic drift, the nondirectional changes in genotypic frequencies among generations due to random chance in small populations, 2) selection, relative differences among genotypes in viability or reproductive success, 3 ) migration, the exchange of genes between popula- tions that differ in genotypic frequencies, and 4) mating, the process mediating the recombination and assortment of genes between generations. If the processes remain intact, then the biological capabilities are expected to be in at least as good a condition in the immediate future as they are now, and would imply a level of sustainability that also is at least as good as now present. While the exact levels and distribution of genetic variation would be expected to have changed during evolutionary history, our central concern is for the processes and the structures necessary to maintain those processes, and not for the present distribution of variation as an end in itself. Mutation is excluded from consideration since we are primarily concerned with relatively short term processes of less than ten generations and with effects of forest interventions that are not likely to significantly change mutation rates.

The effects of various types of biotic and abiotic changes that are evident at the forest stand level are usually felt in more than one of the genetic processes. For example, changes in the abiotic environment can change relative genotypic viabilities but can also affect pollinator behaviour and other inter-species interactions. Because biotic interactions are expected to be more complex in the tropics than in temperate ecosystems, the indirect effects of several, simultaneous environmental changes are expected to be more complex and to operate on a variety of time scales.

\section{Drift}

Populations may be established by few individuals with a limited genetic sample, or may be periodically reduced and lose genes at random. Thus, levels of genetic variation are directly affected by processes of genetic drift and forest practices affect drift. Forest operations or fire may leave few seed or pollen trees, sources of migration may be cut off, and pollinators may have reduced effectiveness, all leading to a reduction in the effective population size $(\mathrm{Ne})$ and to random losses of variation by drift. The number of mating individuals and their reproductive output may be much smaller than the number of adults or number of flowers. Furthermore, inequities in mating frequencies and non-random mating generally further reduce Ne. If the reproductive population remains small, the random drift of alleles will severely reduce genetic variation and future adaptability for several generations.

\section{Selection}

Directed selection favors certain genotypes over others and can therefore maintain population viability and reproductive success through adaptive responses. However, some genes would be lost at higher rates than expected by drift alone, and very strong viability selec- 
tion may reduce population sizes below recuperation levels. Thus, selection can reduce genetic variation, and can also change population averages. Both of these shifts may influence future adaptability. Events that lead to genetically significant selection effects may be unintentional, and a single event such as harvest, could influence changes in several traits in addition to those considered desirable for harvest. Thus, traits such as juvenile growth rates, reproduction age, and pollinator behaviour, can be affected by logging as well as by the timing and design of cut blocks. Furthermore, selection on one trait may also induce genetically correlated changes in other traits and genetic processes. Hence, even simple genetic variation that may affect present and future viability and reproduction.

\section{Mating System}

The mating system that mediates the inter-generational process of evolution is also subject to change due to forest level events. The mating system determines the make up of the male and female mating pools, the extent to which genes are exchanged between individuals (outcrossing rates), and the rates of immigration and emigration. Thus, the levels of heterozygosity, the rates at which vegetative propagation exist, and the exposure of allelic variants to selection, are all affected by changes in the mating system. Forest level events such as logging, thinning, or type conversion, can change population density, pollen and seed dispersal rates and dispersal mechanisms, and timing of flowering, which change the mating system and can increase the effects of mating incompatibilities. Populations that are usually large but suddenly undergo severe reductions may also be exposed to inbreeding depression and loss of viability, reducing their immediate capacity for survival.

\section{Migration}

Changes in the degree of seed and pollen exchange between populations directly affects the divergence between populations and indirectly affects drift within populations. Conversely, high migration rates may also increase local levels of genetic variation. The mating system is also affected by both seed and pollen migration which affects the pool of potential mating partners. Isolation between populations can be affected by the increase or decrease of barriers to exchange between distant populations or by changes in pollen or seed vectors. The form and rates of migration mediate the genetic divergence between partially independent populations of a species (i.e., meta-populations) and affects the distribution of genetic variation. When discontinuities are created by fire or other causes, migration may be altered. The degree of isolation among forest patches will then influence genetic differentiation. For species that are dependent on local extinction and colonization of multiple populations, an additional factor is the adjacency of a competent recruitment pool of immigrants.

\section{Forest Practices that Affect Genetic Dynamics}

Conversely, forest level events due to deliberate forest management and utilization practices or due to inadvertent changes which result from climate change or accidents, can be expected to induce certain kinds of changes to the genetic processes that in turn affect the evolution and sustainability of forests. If management has light impacts or if environmental changes are small, these events may not have the genetic impacts that can be conjectured to occur. However, if they are severe, extensive, or sudden, it would behoove the forest manager to consider their impacts on genetic factors of sustainability. Some of these forest level events are described below and displayed as a matrix in Table 1.

\section{A. Logging}

Logging directly impacts the genetic resource of the commercial species in their population age and density distributions and could directly affect genetic drift in sparsely distributed species. Similarly, the non-commercial competitor or commensalist species, or those in the understorey could be similarly and directly affected, as could forest dependent animal species. If any of these have small populations initially, then drift effects would be enhanced. Directional selection would also be implicated at least for the logged species, but the conversion of the cover type to open growing conditions would also affect the conditions required for survival and reproduction of all species. For the non-commercial species, indirect selection effects can be strong. For isolated populations of any type of species, the response to selection can be strong, and for species with specialized pollen or seed dispersal mechanisms, logging would also be expected to change the mating system. Effects on the seedling generation may be different yet.

\section{B. Grazing}

Grazing would be expected to have a thinning effect on regenerating vegetation and hence could affect genetic drift. It could also affect the understorey vegetation that may also be directly grazed and hence exert a selection effect and changes in the population density of those species, affecting their selection and drift effects. Since grazing may also compact soils and alter stand structure, it may also induce selectively significant environmental changes. 
Table 1. Genetic consequences of different types of forest level events.

\begin{tabular}{|l|c|c|c|c|c|}
\hline & Genetic Drift & Direct Selection & Indirect Selection & Mating System & Migration \\
\hline Logging- Commercial Species & $\mathrm{X}$ & $\mathrm{X}$ & & $\mathrm{X}$ & \\
\hline Logging- Non-commercial Species & $\mathrm{X}$ & & $\mathrm{X}$ & $\mathrm{X}$ & \\
\hline Grazing & $\mathrm{X}$ & & $\mathrm{X}$ & $\mathrm{X}$ \\
\hline Fire & $\mathrm{X}$ & & & $\mathrm{X}$ \\
\hline NTFP - Reproductive & & $\mathrm{X}$ & & & \\
\hline NTFP - Non-reproductive & & $\mathrm{X}$ & & $\mathrm{X}$ & $\mathrm{X}$ \\
\hline NTFP - Whole Individual & $\mathrm{X}$ & & $\mathrm{X}$ & $\mathrm{X}$ \\
\hline Forest Conversion & $\mathrm{X}$ & & & & $\mathrm{X}$ \\
\hline
\end{tabular}

(Each $\mathrm{X}$ indicates the type of forest level event that is likely to change the indicated process, at a level which depends on the specific biological situation and the intensity of utilization.)

\section{Fire}

Intense fire that inflicts heavy mortality and reduces population sizes, would increase drift. By thinning the entire vegetation, some effects of drift may be changed. Migration may actually increase if the migration vectors are abiotic, but may decrease if biotically dependent. Direct selection may only be felt on traits that affect fire resistance, since devastating fires would indiscriminately remove all genotypes.

\section{Harvesting Non-Timber Products}

Harvesting parts of trees or shrubs may involve their reproductive structures such as fruit or seed, and directly reduce the effective size of the pool of reproducing parents. The food derived from some Acacia seeds, and the extractive oils of some Dipterocarp seeds are highly valued and their harvest could substantially increase the effects of genetic drift. However, since these harvest methods are usually environmentally benign, direct selection effects may be minimal except on the reproductive traits themselves. The strongest genetic effects would be felt on the mating system and gene migration dependent on the harvested parts. If non-reproductive parts are harvested, such as leaves for certain medicines, then only indirect selection effects on viability or fecundity of affected individuals could be genetically significant. Only if whole plants are harvested would the effects of reduced population size be genetically significant. For example, whole fern plants may be harvested and used, and while only the growing shoot tip of bamboo or heart of palm may harvested, the whole plant is lost.

\section{Selection of Species}

The effects of forest practices and environmental change can be expected to influence all forest depen- dent species, and since indicators of genetic processes cannot be applied to all species in the forests a system for establishing species priorities for obtaining genetic indicators is needed. Three reasons can be put forward for considering which species to study; 1) utility value - species valued for their known or potential contribution to the economic welfare of the forest users or for their ecological significance as keystone species, 2) existence value - species whose existence per se is valued such as those that are listed due to threat of at least local extinction that if lost, would constitute treaty violations for biodiversity, and 3 ) indicator value species of use as paradigms of a large class of species. In any particular forest management unit, it is expected that the forest level events are known and that the species most directly affected by those events would also be known, but for the sustainability of the forest, all of the genetic resources should be considered.

Species should be considered for genetic study of they fit any of the three categories of value: those that have present or high potential economic significance or have critical ecological significance, those that are listed as being threatened or endangered, and species that are representative of a large class of species that may be ecologically significant if diminished.

\section{Criteria, Indicators and Verifiers}

The primary goal of the workshop and of this report is the development of biological criteria and indicators of sustainable forest management. Since genetic processes and dynamics are not identical with ecological processes and can involve practices to restore genetic variation, genetic criteria for sustainability includes the capacity for restoration as well as selfsustainability. Genetic variation is the sine qua non of evolution and requires that differences in genetic mate- 
rials exist within a species. The level, distribution, and biological significance of the variation is measured in several ways and by several kinds of estimators to which we refer below. We consider only one criterion but two types of indicators, those for the maintenance and conservation of the processes which maintain genetic variation and evolutionary capacities without intensive genetic management intervention, and those that consider the potential capacity for effective genetic management. For each of the indicators of self-sustenance and restoration, we list several verifiers which are the statistics and estimators that can be used as quantifiable guidelines for decision making.

\section{CRITERION : Conservation of the processes that maintain genetic variation.}

Different types of forest use, exploitation, or inadvertent events are likely to affect several of the processes that maintain genetic variation as indicated in Table 1. The processes of genetic change have effects that can be measured in the level and structure of genetic variation and in the processes themselves. These measures can serve as indicators of whether genetic variation is being maintained and the processes of evolution are being maintained. We use 4 indicators of the genetic processes;

1) level of genetic variation,

2) directional change in gene or genotypic frequencies,

3) gene migration between populations, and

4) mating system processes.

As shown in Table 2, one or more indicators can be used for each of the genetic processes.

As discussed in the Introduction, different types of exploitation are likely to affect different processes which act to maintain genetic diversity. One or more indicators are proposed for each of these processes.

To be most useful to forest managers, we can combine the effects of forest level events on genetic processes and the indicators of those processes as given in Tables 1 and 2, to provide managers with tools to warn of changes in genetic processes which arise from particular management practices or by accident. In Table 3 , the indicators are directly linked to some forest uses and other forest level events. For example, logging directly affects population sizes of the harvested species and will increase genetic drift which would be reflected in levels of genetic variation in the residual population. By reducing population sizes of mature trees, changes in the mating system would also have to be anticipated, and could be measured by changes in the rate at which self pollination occurs or in the numbers of partners with which mating is successful. Logging would also bring directional selection pressure on the harvested and other colaterally affected species.

Harvesting of non-timber products, especially of pollen or fruit would not only change the size of the reproductive population which would affect drift and be reflected in the level of genetic variation. This kind of harvesting could also affect directional selection as reflected in changes in gene frequencies, and it would affect the rates of pollen and seed exchange between populations and would directly affect the mating system. These changes could be measured in changes in the rate of inbreeding and in the degree of population differentiation. Harvesting of non-reproductive parts however would not be expected to affect rates of gene flow nor the mating system and hence only monitoring of levels of genetic variance and changes in gene frequencies would be called for.

Other forest level events such as fire, might affect only the genetic processes of drift and gene flow but these process changes may be reflected in changes in the level of genetic variation within populations, the divergence between them, and in the mating system. Whole forest conversions would obviously affect all of the genetic processes and their effects would be reflected in all of the indicators. Grazing on the other hand would only directly involve population sizes and

Table 2. Indicators of genetic processes

\begin{tabular}{|l|c|c|c|c|}
\hline \multirow{2}{*}{ Processes } & \multicolumn{4}{|c|}{ Indicators } \\
\cline { 2 - 5 } & $\begin{array}{l}\text { Levels of genetic } \\
\text { variation are maintained }\end{array}$ & $\begin{array}{l}\text { There is no directional } \\
\text { change in genic/ } \\
\text { genotypic frequencies }\end{array}$ & $\begin{array}{l}\text { There are no changes } \\
\text { in the mating system }\end{array}$ & $\begin{array}{l}\text { There are no changes } \\
\text { in gene migration }\end{array}$ \\
\hline Genetic drift & $\mathrm{X}$ & $\mathrm{X}$ & & \\
\hline Direct selection & $\mathrm{X}$ & $\mathrm{X}$ & & \\
\hline Indirect selection & $\mathrm{X}$ & & $\mathrm{X}$ & \\
\hline Mating & & $\mathrm{X}$ & $\mathrm{X}$ \\
\hline Gene Flow & & & & \\
\hline
\end{tabular}

(As discussed in the Introduction, different types of exploitation are likely to affect different processes which act to maintain genetic diversity. One or more indicators are proposed for each of these processes.) 
Table 3. Indicators of processes that may be affected by forest events

\begin{tabular}{|l|c|c|c|c|}
\hline \multirow{2}{*}{ Modes of Utilization } & \multicolumn{4}{|c|}{ Indicators } \\
\cline { 2 - 5 } & $\begin{array}{l}\text { Levels of genetic } \\
\text { diversity are } \\
\text { maintained }\end{array}$ & $\begin{array}{l}\text { There is no directional } \\
\text { change in genic/ } \\
\text { genotypic frequencies }\end{array}$ & $\begin{array}{l}\text { There are no changes } \\
\text { in the mating system }\end{array}$ & $\begin{array}{l}\text { There are changes } \\
\text { in gene migration }\end{array}$ \\
\hline Logging - Commercial Species & $\mathrm{X}$ & $\mathrm{X}$ & $\mathrm{X}$ & $\mathrm{X}$ \\
\hline Logging - Non-commercial Species & $\mathrm{X}$ & $\mathrm{X}$ & $\mathrm{X}$ & $\mathrm{X}$ \\
\hline Grazing & $\mathrm{X}$ & $\mathrm{X}$ & $\mathrm{X}$ & $\mathrm{X}$ \\
\hline Fire & $\mathrm{X}$ & $\mathrm{X}$ & $\mathrm{X}$ \\
\hline NTFP - Reproductive & $\mathrm{X}$ & $\mathrm{X}$ & $\mathrm{X}$ & \\
\hline NTFP - Non-reproductive & $\mathrm{X}$ & $\mathrm{X}$ & $\mathrm{X}$ & $\mathrm{X}$ \\
\hline NTFP - Whole Individuals & $\mathrm{X}$ & $\mathrm{X}$ & \\
\hline Forest Conversion & & & & \\
\hline
\end{tabular}

(By combining Tables 1 and 2, modes of exploitation can be explicitly linked to indicators through the processes which maintain genetic diversity.)

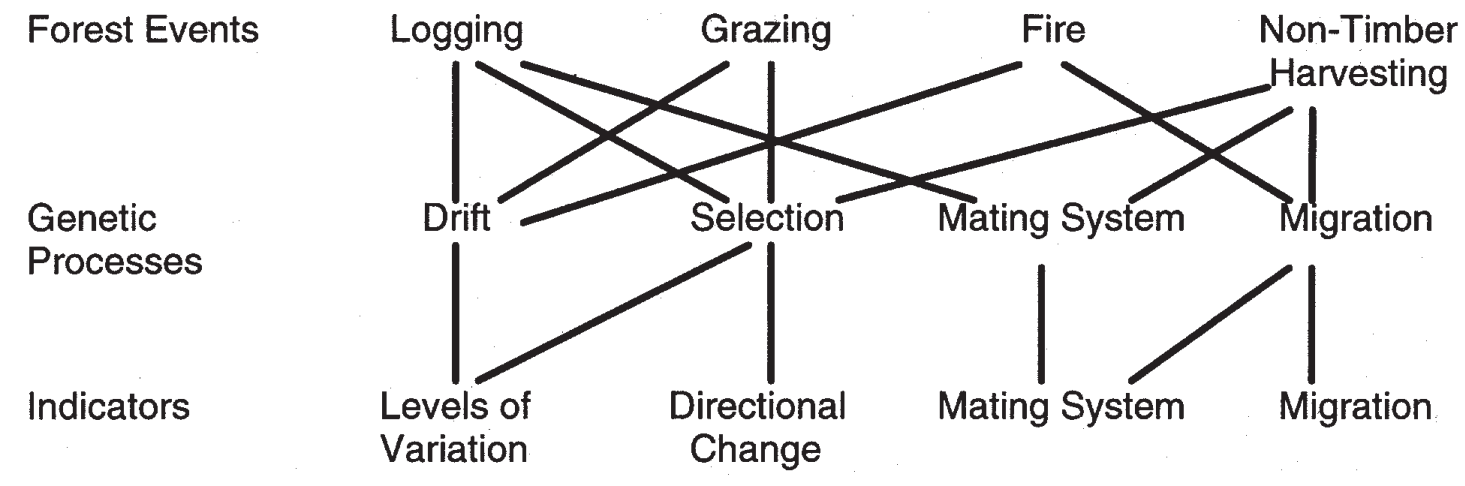

Figure 1. Relationship of forest events to genetic processes and their indicators

drift of the grazed species and this would be reflected in their levels of genetic variation. These relationships are shown in Table 3, and diagrammed in Figure 1.

For each of the indicators, the verifiers may be parameters of the genetic system that directly reflect genetic processes or other factors of the reproductive system, or the verifiers may be estimates of demographic statistics from which we can infer genetic processes. Genetic parameters such as gene frequencies can serve as measures of the level of genetic variation, and differences in frequencies can be used to estimate population differentiation. These data can also be analyzed to estimate parameters of the mating system, such as the outcrossing rate, selection effects on fertility, and migration rates. Changes in population sizes, and age class structure may also reflect changes in the levels and distribution of genetic variation as well as on the mating system.

The different verifiers that can be measured and used for any one indicator, differ widely in the precision with which they estimate the genetic parameters, and in the costs and facilities required to sample and measure them. We list several verifiers for each indicator and in general, expect that for each forest management unit, a feasible set of verifiers can be chosen. The more costly and precise measures would generally be reserved for 
use on only those most critically threatened processes where decisions require high precision, and cruder measures would be used when precision is not needed. However, in some cases, where useful verifiers may be too difficult to use, poor verifiers may be useless or misleading. In such cases, it is preferable to state the lack of data rather than induce a false sense of security. For each indicator, verifiers are listed in the approximate order of precision with the least precise but easiest verifiers to measure, last.

Many if not all of the population genetic parameters, including averages, variances and higher moments, are necessarily measured on and refer to the state of the population rather than directly to population genetic dynamics. Unfortunately, most of the problems involve factors of dynamics and changes in all of the parameters. Sampling and analysis should therefore be directed to ensure that differences are included among populations either spatially or over time and generations and that the appropriate factors such as location, generation, age, density, etc., are identified. This requires that differences between baseline and post-treatment parameters are estimated. If the forest management unit is large, then the distribution of samples at the appropriate scales must ensure that micro- and macro-scale processes are measured. The time scale becomes very important when changes and rates of change are to be assessed. However, it is rarely possible to directly assess changes that occur on evolutionary time scales when forest management decisions have to be made in time scales of months or a few years. Therefore, important differences will often be estimable only by assessing differences among contemporary populations with differences in age or environmental factors, and not by following cohorts over several years or generations. While such compromises may be necessary, research will be necessary to validate the assumptions on which the substitutions of variables are made.

Since variation exists at many gene loci and these loci are partially independent in their evolutionary behaviour, different verifiers can provide more complete information about all of the genetic processes than can single estimators. For example mating system parameters are best measured by verifiers that are not subject to selection on vegetative vigor, whereas parameters of adaptedness to environmental conditions are best measured by verifiers of traits such as growth or phenology that may be heritable. Thus, multiple genetic verifiers are preferred over single verifiers for complex events. Physiological traits would be particularly useful as estimators of adaptability to general environmental variables as well as to stress conditions, but to demonstrate their heritability, expensive and time consuming experimental plantings are usually required. The development of accurate, short-cut procedures would be very valuable for researchers to produce. Many molecular marker techniques such as RFLP's, RAPD's, AFLP's, and SSR's can provide a multitude of data on non-selective processes since they mostly have no physiological effect. Some markers may be associated with physiological effects but finding these markers requires special experimental methods such as used in QTL analyses. Random markers are not likely to show associations in large, predominantly out-crossed species and may be useful only if very large numbers of markers are well dispersed throughout the genome. Some markers have functions such as isozymes and those derived from CDNA probes. However, currently available molecular methods are either too difficult to use in most field conditions, or require such large initial development time, that they would not often be useful. More research is needed to develop the procedures, facilities, and training, before the molecular methods can be useful in all but the most critical and valuable cases. Ideally, a broad mixture of phenotypic, field measures, and multiple locus molecular markers that would sample the whole genome would be available to provide direct genetic process information.

The above indicators consider the genetic processes of each species as if they were independent of the ecosystem on which they have impact and which impacts them. While useful for genetic analysis, the effects of genetic management on the target species on the ecosystems in which they reside must be considered. Forest species will have evolved with different natural histories and would not be equally easy nor equally valuable to sustain in the future. Indicators of the potential for genetic management of species are therefore needed to judge the likelihood that recommended actions could successfully sustain the genetic resource. A genetic "triage" can be useful in this context to assess the potential for restoration and future sustainable management. In addition to the 4 indicators of the genetic processes, we list 2 indicators of management; indicators of the need or desirability of genetic management, and indicators of preventive or remedial action.

\section{INDICATOR 1. Levels Of Genetic Variation}

VERIFIER 1.1 Genic diversity, percent polymorphic loci $(\mathrm{P})$ and related measures of genotypic variance. Measures of average levels of genetic variation at gene loci with high levels of allelic variation. Different measures give weight to different aspects of genotypic and allelic variation. Preferably 
measured on relatively unimpacted cohorts of the target population as well for contrast. A most direct and precise measure but requires expensive and high quality facilities that are rarely now available. (See Yeh, 1996, and Gillet, 1995 for estimators and computer analytical packages.)

VERIFIER 1.2 Effective number of alleles $(\mathrm{Na})$ and related measures of allelic variation but also requires costly laboratory facilities. Measures of levels of gene variation at loci with more than one allele and summarized over loci within gametes. It is also most useful for process indication if measured on cohorts in contrasting conditions. (See Yeh, 1996, and Gillet, 1995 for estimators and computer analytical packages.)

VERIFIER 1.3 Genetic variation as estimated on adaptively significant traits in common garden experiments. Potentially, the most powerful parameter to measure but often difficult to estimate for species in the humid tropics. (See Williams and Matheson, 1994 for design and analysis.)

VERIFIER 1.4 Fixation indices such as Fis, Fst, or Q's and related measures of inbreeding and population differentiation. These estimates can be derived from the data of verifiers $1.1,1.2$, or 1.3, and carry the same constraints for applicability.

VERIFIER 1.5 Numbers of potential parents or sexually mature individuals. After accounting for mortality, fecundity, and fertility differences in sexual contributions, such enumerations can be crude but reasonable estimates of the mating pool. Further research on verifiers 1.1 to 1.4 can provide corrections to this verifier which can be simple and least costly to obtain.

\section{INDICATOR 2:Directional Change In Allele Or Genotype Frequencies}

Selection is the main genetic force that would change gene and genotypic frequencies in a directed fashion. It operates on phenotypic traits exhibited by individuals, and directionally affects genotypic frequencies to the extent that those traits are heritable. In systems of selective harvesting or breeding, conscious selection may be assumed, but in many cases, indirect selection effects may also change gene and genotypic frequencies. These may be due to the complexities of the selective process such as logging which may selectively remove understorey individuals of a particular size or form, or may be due to complexities of the genetic system where genetic changes in one trait like size, may affect changes in a correlated trait like age of reproduction.

VERIFIER 2.1 Differences in genotypic frequency distribution among cohorts. These differences may be measured as changes in levels of heterozygosity, for example, in cases where inbred individuals are eliminated. If generational changes cannot be sampled, then the changes may be observed among age classes in the same stand if the cohorts were initially similar. In some cases, selection on a trait will result in directional changes in genotypic frequencies at some marker loci if there is close linkage and stochastic association between the marker and the selected loci. It should be noted that the lack of detectable changes in gene frequencies does not prove an absence of selection. These measures are the most technically demanding, and often provide weak tests of selection, but are direct measures of genetic change.

VERIFIER 2.2 Differences in phenotypic frequency distributions among cohorts. These differences would be expected to be most strongly evident in traits with high heritability and those under direct selection pressure. Other traits that may be indirectly affected but of potential significance for future adaptations may be less obviously affected but should also be observed. Differences may be observed in several different statistical moments of the distribution, each reflecting different types of selection. These differences are most powerfully measured in common garden experiments as for verifier 1.3 , but inferences can be drawn on the basis of paired stands or cohorts.

VERIFIER 2.3 Differences in age class distribution. Differences in demographic parameters are indirect measures of effects that may be present at the genic level, and may be the easiest data to obtain. Under conditions of minimal disturbance or steady state environments, an equilibrium age class distribution may be attained, and if disturbed, would portend gene frequency changes as well. Hence relatively undisturbed cohorts may provide contrasts or if obvious imbalances exist such as an absence of a seedling recruitment class, directional selection may be presumed. In some secondary successional stages, and other non-equilibrium ecosystems, distributional differences are expected. While such shifts and any concomitant genetic changes may not imply threats of extinction, the change is important to track. 


\section{INDICATOR 3: Migration}

Genetic migration between populations is a genetic process that is the obverse of genetic isolation. Whereas isolation leads to genetic drift among populations, migration tends to allow genes to be shared among populations and to hold frequencies to a species average. Physically, it also allows for apparently isolated individuals to participate in a general mating pool and if mediated by seeds, to colonize habitats. The structure of genetic variation within and between populations depends on the balance between forces of diversification and homogenization, and changes may be neither helpful nor harmful for future adaptability, but would be important to monitor.

VERIFIER 3.1 Genetic differentiation between populations as measured on cohorts can be measured by different statistics such as Fst, Gst, d, Q, Dj, and others. High levels of differentiation indicate low levels of migration but may be confounded with divergent selection, and drift. These parameters may be estimated with molecular markers or more strongly by phenotypic traits, with both requiring substantial technical input.

VERIFIER 3.2 Change in pollen dispersal distance or numbers of potential mates that would impact on genetic variation of progenies. The effective distance may be affected by changes in the guild of pollinator species as well as by physical distance between mature adults.

VERIFIER 3.3 Changes may similarly be observed in seed dispersal distances by physical or biotic factors affecting seed dispersal guilds.

VERIFIER 3.4 Changes in degree of physical isolation by loss of neighbors or by stronger physical or biotic barriers to pollen or seed dispersal.

VERIFIER 3.5 Changes in the spatial aggregation of divergent mating types such as segregation of age classes, or mating behaviour.

\section{INDICATOR 4: Mating System}

The mating process is the bridge between generations and hence mediates evolution. The mating system involves the levels of fecundity and fertility, propensities for inbreeding vs. outbreeding, and unequal or nonrandom mating associations. It is the primary determinant of how genetic variation is partitioned among individuals (heterozygosity) and hence, of how much inbreeding depression will be expressed. It is affected by individual sexuality, phenology, specific compatibility relationships between potential mates and is subject to selection. Measures of pollen flight and numbers of reproductive organs have proven to be difficult to obtain in the field and have failed to accurately predict the genotypic structure of the resulting seedling pool.

VERIFIER 4.1: Changes in outcrossing rate reflect increases or avoidance of inbreeding. The mean percentage of outcrossed progeny can be estimated on open-pollinated families with allozyme or other markers that require laboratory and field storage capabilities. The same data can also be used to estimate the fixation index (Fis) and other statistics that reflect non-random mating.

VERIFIER 4.2: Changes in sex ratio or effective sexuality reflect changes in mating success. Direct counts of reproductive organs indicates the potential mating pool and cohort pairs provide contrasts to indicate change.

VERIFIER 4.3: Changes in pollinator abundance or composition indicates potential changes in male gamete dispersal.

VERIFIER 4.4: Changes in the density of the potential parental pool reflect changes in mating success or in numbers and synchrony of reproductive behaviour reflect changes in specific parental associations beyond normal fluctuations.

VERIFIER 4.5: Changes in proportion of filled seed or germination percent reflect changes in mating success but do not indicate the number or distribution of mates.

\section{Indicators of Preventive or Restorative Actions}

The potential of the genetic system for recovery and selfsustenance or for management interventions of one sort or another would indicate that sustainable forestry is in fact biologically possible. However, as a result both of evolutionary history and past management actions, the genetic diversity of different species will be neither equally easy nor equally valuable to manage. It may therefore be a useful exercise in determining management priorities to invoke a process of "genetic triage" in which the potential for recovery and future sustainable management of the genetic resource is assessed. This requires a set of parameters which can act as indicators of, 
firstly, what specific actions are required to effect genetic recovery and, secondly, whether the proposed actions are operationally feasible or not.

\section{INDICATOR 5: Actions required to protect or restore genetic diversity}

This indicator will take the form of critical threshold values of indicators 1 to 4 (and their verifiers). For example, if a verifier of levels of genetic variation (Indicator 1) indicates that the current state of genetic variation is lower than expected or acceptable, a specific restorative action may be indicated. In a logging situation, the types of restorative actions indicated may be to raise the diameter limit, or to lengthen the cutting cycle. If, however, the verifier used for Indicator 1 detects a more serious shortfall in genetic variation, more intensive restorative actions may be indicated, for example collection of material from other sources and enrichment planting. Thus, there may be more than one threshold value for verifiers of Indicators 1-4, which indicate different intensities of protective or restorative action.

These threshold values are currently not known. How low levels of genetic variation may fall, or how far the rate of inbreeding increases before deleterious effects occur is not known for any species. This is an area requiring substantial additional research effort (see section below on Research Priorities).

\section{INDICATOR 6: Feasibility of protective or restora- tive actions}

While indicator 5 may be useful in suggesting specific actions to be undertaken, such actions may not be operationally feasible for a variety of reasons. To take the logging example used in the previous section, if an increase in the diameter limit is indicated as a suitable restorative measure for a loss of genetic variation, verifiers of the practicality of such an action may include the maximum diameter reached by that species, the availability of less affected species (impacting the overall economic returns of the logging operation), etc. For a more serious shortfall in genetic variation, indicating the need for enrichment planting, verifiers would include the presence of alternative seed sources, and the existence of nursery and planting techniques for that species. Verifiers for this Indicator obviously require further elaboration.

\section{Decision Support Systems}

As mentioned earlier, it is not economically feasible to expect all Indicators to be assessed in every situation, or that the most sophisticated verifiers be always used. Depending on the uses made of the forest, some indicators may not need to be assessed at all, or need only be assessed using cheap and approximate verifiers. Other indicators will be at very great risk - for example, changes to the effective mating system due to collection of reproductive structures for non-timber forest products. Table 3 provides a crude form of a decision support system in that it permits a manager, who may have little or no knowledge of genetics, to determine which indicators should be assessed, based only on a knowledge of the activities going on in the forest. To be most useful, however, a much more sophisticated decision support system is required. If collection of reproductive structures for non-timber forest products is an activity in the forest, Table 3 indicates that all of Indicators 1-4 should be assessed. But which one is the most critical - if financial resources only permit assessment of two or three of the indicators, which ones should they be? And what level of verifier should be used - is it better to adopt cheaper verifiers to allow assessment of more indicators, or is one or more of the Indicators so critical that no cost saving can be permitted?

The same kind of questions are applicable to Indicators 5 and 6 . Several restorative actions may be indicated, but which one is the most important? Also, while raising the diameter limit may be biologically feasible, it may have serious repercussions on nongenetic Indicators, such as those dealing with economic feasibility. Therefore, an effective decision support system must take account not only of genetic Indicators in isolation, but of all biological, economic, and social indicators. With limited resources, is it more important to assess Economic Indicator x or Genetic Indicatory?

What the manager requires is a dual Decision Support System. The first, or "front-end" system allows him/her to input basic descriptive data concerning the forest management unit, and to have generated a prescription for assessment of sustainability, by identifying and ranking the important Indicators and Verifiers across all disciplines. The second component of the system allows the manager to enter the results obtained from the Indicators and Verifiers identified as being important, and to receive a prescription of what preventative or restorative actions are necessary - again, ranked in order of importance - so as to maintain or achieve overall biological, economic, and social sustainability.

Clearly, the sophistication of such a Decision Support System is beyond the capacity of a simple rule set as depicted in Table 3. It needs to be based on computer models of the entire forest system, 
biological, economic, and social Indicators. This requires a major effort in multidisciplinary research and modelling.

\section{Research Priorities}

Since genetic processes are usually not directly observable, and those parameters that are most precise and informative are often expensive to develop and to use, two problems exist that can be relieved by further research. Research to better predict the relationships between ecological or demographic factors and genetic processes would allow us to predict what types and intensities of forest level events portend significant changes in genetic parameters. Better understanding of those relationships would also allow us to predict the ecological effects that changes in the genetic system would be reasonable to infer. The relationships described by the matrices in Tables 1,2, and 3, are very crude and their refinement to include levels of intensity would be very useful in a decision support system. Furthermore, with more precise information on the ecological-genetic interface, more precise surrogate measures can be developed and more efficient measurements can be taken. Opportunities for genetic research should be sought where ecological measurements are already planned. This would provide the forest manager with more options for choosing measures that are appropriate and available for specific forest conditions.

\section{Specific Research Areas}

1. Effect of environmental, biotic, demographic changes on genetic processes and parameters, and their effects on different types of gene markers.

2. Effects of genetic variables on demographic and ecological processes.

3. Predictive criteria for species that are vulnerable to genetic change.

4. Defining critical levels of genetic verifiers that call for intervention actions.

5. Methods for estimating phenotypic genetic variation in-situ.

6. Determining utility of surrogate measures such as changes in pollinator guilds that actually change outcrossing rates.

7. Develop indirect estimators of genetic parameters that are more easily measured in the field such as correction factors for adult numbers or contrasts between cohort population frequencies and variances.

8. Generate generally applicable base-line genetic data on population structure and levels of variation and genetic processes for tropical tree species that exist in different successional or dispersal states. 


\section{REFERENCES}

Gillet, E. M. 1994. Genetic Structures from Electrophoretic Data, Version 1.0. Abteilung für Forstgenetik und Forstpflanzenzüchtung, Universität Göttingen, Büsgenweg 2, D37077 Göttingen, Germany.

Prabhu, R., Colfer, C.J.P., Venkateswarlu, P., Tan, L.C., Soekmadi, R. and Wollenberg, E. (1996) Testing Criteria and Indicators for Sustainable Management of Forests: Final Report of Phase I. CIFOR, P.O. Box 6596, Jakarta 10065, Indonesia (In preparation).

Williams, E. R. and Matheson, A. C., 1994. Experimental Design and Analysis for use in Tree Improvement. CSIRO Information Services, 314 Albert Street, E Melbourne, Victoria 3002, Australia.

Yeh, F. 1996. Popgene Version 1.0. Microsoft Window-based Software for Population Genetic Analysis. Forest Genetics Laboratory, University of Alberta, Edmonton, Alberta, Canada.

\section{Workshop Participants}

Dr. Gene Namkoong (University of British Columbia, Canada)

Dr. Tim Boyle (CIFOR, Indonesia)

Dr. Hans-Rolf Gregorius (University of Göttingen, Germany)

Dr. Hélène Joly (CIRAD-Forêt, France)

Dr. Outi Savolainen (University of Oulu, Finland)

Dr. Wickneswari Ratnam (Forest Research Institute of Malaysia, Malaysia)

Dr. Andrew Young (CSIRO, Australia) 